\title{
Atypical cerebral dominance in Down's syndrome
}

\author{
DANIEL J. WEEKS \\ Lakehead University, Thunder Bay, Ontario, Canada \\ and \\ DIGBY ELLIOTT \\ McMaster University, Hamilton, Ontario, Canada
}

\begin{abstract}
Recent research in which a number of procedures have been used to assess cerebral specialization in individuals with Down's syndrome (DS) is summarized. We propose a model of cerebral specialization that has as its core feature the biological dissociation between speech perception and the production of speech and other complex movements. Initial tests of the model are discussed with respect to some of the cognitive deficits experienced by DS individuals.
\end{abstract}

Over the past several years, a number of attempts have been made to determine the manner in which the Down's syndrome (DS) karotype influences cerebral development to produce intellectual and information-processing deficits in the DS population (e.g., Pipe, 1983, 1988; ZekulinHartley, 1978, 1981, 1982). As a result, several suggestions have been made with regard to a syndrome-specific reversal of cerebral function (see, e.g., Hartley, 1981, 1982). The evidence for such a reversal follows primarily from the use of a dichotic listening paradigm.

Recently, Pipe (1988) examined the evidence for a relation between atypical cerebral laterality and deficient cognitive performance in retarded individuals and concluded that there is "strong evidence of atypical lateralization for some retarded groups such as those with Down's syndrome"' (p. 343). However, Pipe lamented that " unfortunately, it is not presently clear how, or even whether, this atypical pattern of asymmetries might shed light on the cognitive disabilities of either autistic or Down's syndrome children' (p. 345).

Previously, we (Elliott, Weeks, \& Elliott, 1987) critically evaluated the various dichotic listening findings and, like Pipe (1988), we concluded that "although the argument continues as to whether individuals with Down syndrome have a reversed cerebral specialization for speech sounds, it is clear that they do not exhibit typical rightear-left-hemisphere dominance in tests of dichoticlistening"' (p. 265). As part of our evaluation, we determined that evidence obtained in our lab makes any simple model of reversed cerebral specialization in DS in-

\footnotetext{
We would like to thank all of the colleagues we have had the good fortune to have worked with on the various experiments reported here: Heather Carnahan, Jackie Edwards, Connie Elliott, Ruth Jones, Tim Lee, and Sue Lindley. Thanks also go to Robert Proctor for comments and suggestions and to Chris Wreszczak for preparing the manuscript. Many of the experiments discussed in this paper were funded by the Ontario Mental Health Foundation. As well, the authors acknowledge the support of the Natural Sciences Engineering and Research Council of Canada. Correspondence should be addressed to Digby Elliott, Motor Behaviour Lab, Department of Physical Education, McMaster University, Hamilton, Ontario L8S 4K1, Canada.
}

dividuals untenable (Edwards \& Elliott, 1987, 1989; Elliott, 1985, 1990; Elliott, Edwards, Weeks, Lindley, \& Carnahan, 1987; Elliott \& Weeks, 1990; Elliott, Weeks, \& Jones, 1986). Because dichotic listening is only one index of cerebral dominance, in our work we have used several other procedures to assess lateralization.

Our initial work focused on manual asymmetries. ${ }^{1}$ The underlying premise behind this work was that "hand differences in the performance of specific motor tasks are related to the differential ability of the two hemispheres in processing certain types of information" (Elliott, Weeks, \& Elliott, 1987, p. 266). Specifically, superior left-hand performance for spatial-type tasks is often attributed to a right-hemisphere dominance for spatialinformation processing. Alternatively, right-hand superiority on sequential-type tasks (such as rapid fingertapping) is often attributed to left-hemisphere dominance for sequential-information processing.

Although we (Elliott, 1990; Elliott \& Weeks, 1990) have been unable to find left-hand advantages for tactile matching tasks or spatial orientation judgments (cf. Benton, Varney, \& de Hamsher, 1978; Witelson, 1974; see also Hannay \& Smith, 1979; Jones \& Elliott, 1988) for either DS or non-DS individuals, we have been successful in finding manual asymmetries in the organization and execution of sequential movement. In a finger-tapping study (Elliott, Weeks, \& Jones, 1986), DS individuals exhibited the same right-hand variability advantage as nonretarded subjects did. Right-hand consistency in finger-tapping has been attributed to a left-hemispheric superiority for the modulation of force (Todor \& Smiley, 1985) and has been thought to reflect the special role of the left hemisphere in the control of movement (Roy \& Elliott, 1986).

As an adjunct to examining simple performance asymmetries, we have also considered transfer of training between the hands. Employing the general logic of Taylor and Heilman (1980), we (Elliott, 1985; Edwards \& Elliott, 1989) examined intermanual transfer in DS and control subjects trained to perform rapid sequencing tasks with the right or left hand. Training improved performance for both hands, but transfer of training was asym- 
metric. For both DS and normal control subjects, transfer from the left hand to the right hand was greater than transfer in the reverse direction. In keeping with Taylor and Heilman's (1980) suggestion, we interpret these data as indicating that DS persons are left-hemisphere-dominant for movement sequencing, as are normals. Thus, our manual asymmetry studies place in doubt any simple model of reversed cerebral specialization in DS persons.

In another study of cerebral specialization for speech production (Elliott, Edwards, et al., 1987), DS subjects were required to finger-tap with the left or right hand while speaking. Typically, for right-handers, speech disrupts right-hand performance more than left-hand performance (Kinsbourne \& Cook, 1971). This is due to the fact that the left hemisphere is responsible for both speech and right-hand motor control (i.e., within-hemispheric interference, see Kinsbourne \& Hicks, 1978). Once again, we found similar patterns of specialization in DS subjects and nonretarded control subjects (Elliott, Edwards, et al., 1987). Although females were somewhat less lateralized than males, both DS and control subjects overall showed a greater right-hand as opposed to left-hand performance decrement from concurrent speech (see also Harris \& Gibson, 1986, and see Elliott, Weeks, Lindley, \& Jones, 1986 , for a discussion of gender differences). These results suggest that, like most right-handers, right-handed DS persons are left-hemisphere-specialized for speech production (see Carnahan, Elliott, \& Lee, 1986). Again, our work provides evidence against any simple model of reversed cerebral dominance in DS persons.

Although Pipe (1988) noted that dichotic listening studies provide "strong evidence" of atypical lateralization in DS individuals, she concluded rather pessimistically that it is uncertain whether these findings can contribute to an understanding of the cognitive disabilities of DS individuals. Much of the evidence examined by Pipe (1988), primarily from dichotic listening studies, served as the starting point for our research efforts. However, the methodological problems (Tannock, Kershner, \& Oliver, 1984) that preclude simple interpretation of dichotic listening studies prompted us to examine other ways in which to assess cerebral specialization as an adjunct to the dichotic listening work. As a consequence, our interpretation of that literature, together with our empirical work, has culminated in a dissociation model of cerebral organization in DS individuals (Elliott, Weeks, \& Elliott, 1987).

The core feature of the model is the suggestion that DS persons process speech perception with the right hemisphere but organize complex movement control, including speech production, with the left hemisphere. This dissociation of speech perception and speech production has potential for addressing a number of the cognitive deficits experienced by DS persons. In our most recent research, we have been concerned with the practical implications of the model for both performance and learning (Elliott, Gray, \& Weeks, 1991; Elliott \& Weeks, 1990; Elliott, Weeks, \& Gray, 1990).
First, the model predicts that DS persons will likely be deficient on any tasks that demand both the perception and the production of speech. We would argue that many of the sequential language deficits associated with DS may be related to the biological dissociation of structures responsible for speech perception and speech production. Thus, whereas these systems typically overlap in the normal population, their dissociation may contribute to many of the language-related deficits exhibited by the DS population (Ashman, 1982; Gibson, 1975; Hartley, 1982). Second, if speech perception and the control of sequential movement are indeed supported by different hemispheres, then DS persons will exhibit deficiencies in tasks that involve both speech perception and the production of limb movements. In our most recent work, we have attempted to test the latter prediction.

In an initial study (Elliott \& Weeks, 1990), DS and undifferentiated mentally retarded control subjects completed a simple apraxia test battery. The test battery assesses movement proficiency and involves the making of oral and limb movements to verbal or visual cues provided by the experimenter. In agreement with the model, we observed a performance advantage favoring the visual cue condition for the DS subjects. In contrast, the control group exhibited equivalent performance across both cue conditions.

In a second experiment (Elliott \& Weeks, 1990), we cued a series of hand movements either visually or verbally. Whereas nonretarded adults performed equally well in the visual and verbal conditions (i.e., in terms of time to complete the movement series), DS adults exhibited a distinct advantage when the movement sequence was defined visually. This finding is consistent with the predictions of our model, because it suggests that DS persons have relative difficulty when movements must be organized according to a verbally defined sequence.

The dissociation between speech perception and movement organization proposed in the model may explain some of the specific information-processing problems experienced by these individuals. Studies indicate that, when compared with non-DS individuals of a similar mental age, DS persons perform relatively poorly on tasks that require vocal imitation (Mahoney, Glover, \& Finger, 1981), auditory sequential memory (Marchell \& Armstrong, 1982), and sequential language processing (Ashman, 1982). Moreover, persons with DS exhibit performance advantages when they are compared with control subjects matched for mental age on tasks that involve visual pattern discrimination and visuomotor ability (Silverstein, Legutki, Friedman, \& Takayama, 1982). Interestingly-and as our model predicts-the tasks on which DS persons show disadvantages involve both speech perception and speech production, whereas the other tasks do not. Thus, some of the expressive language and movement problems exhibited by DS individuals may be the result of a dissociation between speech perception and brain mechanisms responsible for sequential movement production. Moreover, if systematic patterns of deficit can 
be found in one group of mentally retarded persons, a similar neuropsychological approach may prove fruitful with other groups and/or individuals.

In sum, we believe that there has been considerable progress toward understanding cerebral specialization in individuals with DS, from both a theoretical and a practical standpoint. Moreover, our optimism for further progress remains consistent with our previous research mandate (Elliott, Weeks, \& Elliott, 1987):

Research in this important area should continue, because an understanding of cerebral organization in this population will not only help us appreciate information-processing deficits specific to the syndrome, but it may provide us with important avenues of research related to the genetic underpinnings of brain organization. (p. 269)

\section{REFERENCES}

Ashman, A. F. (1982). Coding strategic behavior and language performance of institutionalized mentally retarded young adults. American Journal of Mental Deficiency, 86, 627-636.

Benton, A. L., VARNEY, N. R., \& DE HAMSHER, K. S. (1978). Lateral differences in tactile directional perception. Neuropsychologia, 16, 109-114.

Carnahan, H., Elliott, D., \& Lee, T. D. (1986). Dual-task interference between speaking and listening and a unipedal force production task. Neuropsychologia, 24, 583-586.

EDWARDs, J. M., \& EluotT, D. (1987). The effect of unimanual training on contralateral motor overflow in children and adults. Developmental Neuropsychology, 3, 299-309.

Edwards, J. M., \& ElliotT, D. (1989). Asymmetries in intermanual transfer of training and motor overflow in adults with Down's syndrome and nonhandicapped children. Journal of Clinical \& Experimental Neuropsychology, 11, 959-966.

EluotT, D. (1985). Manual asymmetries in the performance of sequential movement by adolescents with Down syndrome. American Journal of Mental Deficiency, 90, 90-97.

EluotT, D. (1990). Movement control and Down's syndrome: A neuropsychological approach. In G. Reid (Ed.), Problems in movement control (Advances in Psychology Series No. 74, pp. 201-216). Amsterdam: North-Holland.

Elliott, D., Edwards, J. M., Weeks, D. J., Lindley, S., \& CarNAHAN, H. (1987). Cerebral specialization in young adults with Down syndrome. American Journal of Mental Deficiency, 91, 480-485.

Elliott, D., Gray, S., \& WeEKS, D. J. (1991). Verbal cuing and motor skill acquisition for adults with Down syndrome. Adapted Physical Education Quarterly, 8, 210-220.

ElurotT, D., \& WeEKS, D. J. (1990). Cerebral specialization and the control of oral and limb movements for individuals with Down's syndrome. Journal of Motor Behavior, 22, 6-18.

Elliott, D., WeEKs, D. J., \& Elliott, C. (1987). Cerebral specialization in individuals with Down syndrome. American Journal on Mental Retardation, 92, 263-271.

EllotT, D., WeEKS, D. J., \& GRAY, S. (1990). Manual and oral praxis in adults with Down's syndrome. Neuropsychologia, 28, 1307-1315.

Elliott, D., WeEKs, D. J., \& JoNEs, R. (1986). Lateral asymmetries in finger-tapping by adolescents and young adults with Down syndrome. American Journal of Mental Deficiency, 90, 472-475.

Elliott, D., Weeks, D. J., Lindley, S., \& JoNes, R. (1986). Sex differences in dual-task interference between speaking and a manual force production task. Perceptual \& Motor Skills, 62, 3-8.

GiBson, D. (1975). Chromosomal psychology and Down's syndrome (mongolism). Canadian Journal of Behavioural Science, 7, 167-191.

HanNaY, H. J., \& SMITH, A. C. (1979). Dichaptic perception of forms by normal adults. Perceptual \& Motor Skills, 49, 991-1000.

HARRIS, A., GiBSON, D. (1986, June). Asymmetries in language processing and syndrome specificity in the mentally handicapped. Paper presented at the Canadian Psychological Association Annual Meeting, Toronto.
HaRTLeY, X. Y. (1981). Lateralisation of speech stimuli in young Down's syndrome children. Cortex, 17, 241-248.

HARTLEY, X. Y. (1982). Receptive language processing of Down's syndrome children. Journal of Mental Deficiency Research, 26, 263-269.

JoNES, R., ElLIOTT, D. (1988). Intra- and interhemispheric integration of tactual and visual spatial information. Bulletin of the Psychonomic Society, 26, 229-231.

Kinsbourne, M., Cook, J. (1971). Generalized and lateralized effects of concurrent verbalization on a unimanual skill. Quarterly Journal of Experimental Psychology, 23, 341-345.

Kinsbourne, M., \& Hicks, R. E. (1978). Functional cerebral space: A model for overflow, transfer and interference effects in human performance. In J. Requin (Ed.), Attention \& Performance VII (pp. 345362). New York: Academic Press.

Mahoney, G., Glover, A., \&inger, I. (1981). Relationship between language and sensorimotor development of Down syndrome and nonretarded children. American Journal of Mental Deficiency, 86, 21-27.

Marchell, M. M., \& Armstrong, V. (1982). Auditory and visual sequential memory of Down syndrome and nonretarded children. American Journal of Mental Deficiency, 87, 86-95.

MILNER, B. (1975). Psychological aspects of focal epilepsy and its neurosurgical management. In D. P. Purpura, J. K. Penry, \& R. D. Walter (Eds.), Advances in neurology (Vol. 8, pp. 299-391). New York: Raven Press.

PIPE, M. E. (1983). Dichotic-listening performance following auditory discrimination training in Down's syndrome and developmentally retarded children. Cortex, 19, 481-491.

PIPE, M. E. (1988). Atypical laterality and retardation. Psychological Bulletin, 104, 343-347.

Roy, E. A., ELlotr, D. (1986). Manual asymmetries in visually directed aiming. Canadian Journal of Psychology, 40, 109-121.

Silverstein, A. B., Legutki, G., Friedman, S. L., * Takayama, D. L. (1982). Performance of Down syndrome individuals on the Stanford-Binet intelligence scale. American Journal of Mental Deficiency, 86, 548-551.

Tannock, R., Kershner, J. R., \& Ouver, J. (1984). Do individuals with Down's syndrome possess right hemisphere language dominance? Cortex, 20, 221-231.

Taylor, H. G., Heilman, K. M. (1980). Left-hemisphere motor dominance in right-handers. Cortex, 16, 587-603.

Todor, J. L., SMiley, A. L. (1985). Performance differences between the hands: Implications for studying disruption to limb praxis. In E. A. Roy (Ed.), Neuropsychological studies of apraxia and related disorders (pp. 309-344). Amsterdam: North-Holland.

Witelson, S. F. (1974). Hemispheric specialization for linguistic and nonlinguistic tactual perception using a dichotomous stimulation technique. Cortex, 10, 3-17.

ZexUlin-HARTLEY, X. Y. (1978). Hemispheric asymmetry in Down's syndrome children. Unpublished doctoral dissertation, University of Toronto.

ZeKulin-Hartley, X. Y. (1981). Hemispheric asymmetry in Down's syndrome children. Canadian Journal of Behavioural Science, 13, 210-217.

ZekUlin-Hartley, X. Y. (1982). Selective attention to dichotic input of retarded children. Cortex, 18, 311-316.

\section{NOTE}

1. In our studies, we have examined only right-handed DS subjects, because left-hand preference does not by itself predict a particular pattern of cerebral specialization (Milner, 1975) in non-DS persons. We are aware that the relationship between hand preference and cerebral specialization in persons with DS may be completely different than it is for non-DS persons. Regardless, it seems reasonable first to attempt to understand cerebral specialization in right-handed persons with DS. On a preference test that involves writing, throwing, eating soup, and hammering, we have found that between $85 \%$ and $90 \%$ of the DS adolescents and adults available to us demonstrate consistent right-hand preference. 